\title{
STUDY AND ANALYSIS ON SOUND ABSORBING AND NOISE REDUCING PERFORMANCE OF TIMBER CONSTRUCTION WALL BASED ON ACOUSTIC SPIRAL MATASURFACE
}

\author{
Haiyan Fu, Xinyue Zhao, Patrick Adjei, Zheng Wang, Xiaoli Wu \\ Nanjing Forestry University \\ China \\ (Received June 2020)
}

\begin{abstract}
Based on acoustic spiral metasurface, a spiral structural layer was designed to apply to timber construction interior wall. The sound absorption coefficient was measured by impedance tube method and compared with Helmholtz resonance structural layer, solid structural layer and air layer in traditional wall. The results show that the combination of the spiral structural layer and the wall can optimize the sound absorption performance of the wall in the medium and low frequency. Without reducing the overall sound-absorbing performance of the wall, can achieve perfect sound absorption in some medium and low frequency sound bands.
\end{abstract}

KEYWORDS: Acoustic metasurface-based, timber construction, sound absorption performance, interior wall, spiral metasurface.

\section{INTRODUCTION}

In recent years, due to the national attention to environmental protection and energy conservation, green buildings (Haitao et al. 2019, Azkorra et al. 2015) are more and more popular. Timber or bamboo (Xin et al. 2019, Xin et al. 2020, Cheng et al. 2019) construction not only meets people's basic requirements for buildings, but also meets people's requirements for living environmental comfort and protection, as well as energy saving environment (Ying-Yang et al. 2018, Valachova et al. 2019). The application of this phenomenon is more extensive in China. The timber construction has advantages in physical and mechanical properties, but it still has shortcomings in low frequency noise reduction and sound absorption in wall structure. Generally, in the case of relatively low surface density, it is difficult to calculate the weight of sound insulation $R_{W}$ (weighted sound reduction index) to reach more than $50 \mathrm{~dB}$, or even less than $40 \mathrm{~dB}$. In the low frequency region of the sound frequency band $(100-500 \mathrm{~Hz})$, acoustic resonance phenomenon is prone to occur, which affects the overall acoustic performance of the wall (Caniato et al. 2017). Previous studies have generally improved the sound-absorbing 
properties of walls by changing the internal materials of walls, such as reasonably filling pores with sound-absorbing materials (Zhong-Bin et al. 2015), using composites with high sound-absorbing properties (Bo-Seung et al. 2016, Cao-Ping 2006), or changing the spacing and sizes of wooden keel (Belakroum et al. 2017, Ljunggren et al. 2011). But in the actual control, through these methods and measures, the effect of sound absorption and noise reduction of the wall is not significantly actualize, especially with low and medium frequency noise. Low frequency noise has the characteristics of strong penetration, slow attenuation, long propagation. And low-frequency noise will have a bad impact on the functionalities of some vital organs of the human body. In acoustics, a variety of resonance structures can be used for sound absorption, but the most widely used structure is a Helmholtz resonator. Acoustic metasurface plays a significant role in solving low-frequency vibration and noise reduction (Zhu et al. 2019, Ali et al. 2020, Krupali et al. 2020). Based on the acoustic metasurface, researchers studied the spiral acoustic metasurface, a perfect low-frequency sound absorber (Huang et al. 2018, Li et al. 2016, Huang et al. 2019, Assouar et al. 2018). The purpose of this experimental study is to solve the problem of poor acoustic performance in timber construction. To improve the acoustic performance of wall structure of timber construction, the acoustics metasurface structure combined with wall structure of timber construction. Through optimizing the exterior structure of wall, it is improved the wall of sound absorption performance and the overall structure of the sound insulation performance.

\section{MATERIAL AND METHODS}

\section{Material}

Design principle

As shown in Fig. 1, the spiral structural layer is composed of a perforated plate and a coplanar spiral chamber.

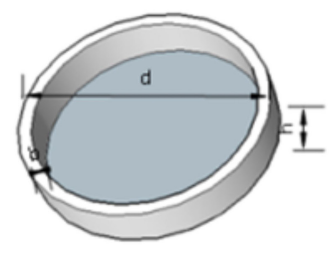

Cavity structure(K)

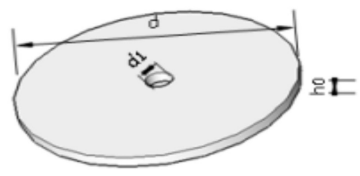

Perforated panel1 $(\mathrm{d} 1=16)$

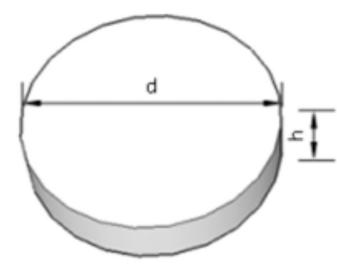

Solid structure(S)

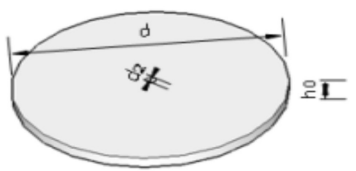

Perforated panel2 ( $\mathrm{d} 2=8$ )

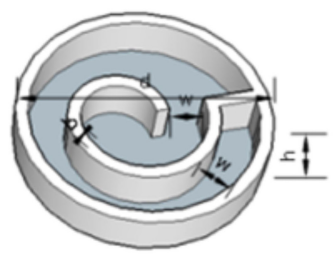

Spiral structure(M)

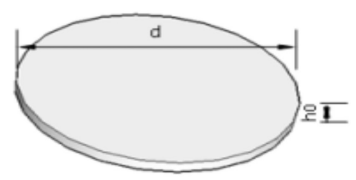

Solid

*Note: The solid structure and solid are the traditional structure (control group).

Fig. 1: Design drawing of structural layer.

The plane sound wave propagates along the "z" direction. The sound wave enters the coplanar spiral chamber through the hole in the perforated plate. It is completely reflected 
at the end of the spiral chamber and then emitted from the hole, forming a reflected wave. The structure is actually a resonant sound absorption structure composed of a perforated plate and a cavity behind the plate. Its acoustic impedance $Z_{\mathrm{s}}$ is formed by the equivalent acoustic resistance $x_{\mathrm{s}}$ and acoustic reactance $y_{\mathrm{s}}$ of the perforated plate and the coplanar spiral cavity in series (Li et al. 2016, Dah-You et al. 1998, Ingard et al. 1953), which can be expressed as follows:

$$
z_{s}=x_{s}+i y_{s}
$$

When the plane sound wave is vertically incident, the sound absorption coefficient of the perforated plate absorber is:

$$
\alpha=\frac{4 x_{s}}{\left(1+x_{s}\right)^{2}+y_{s}^{2}}=\frac{4 x_{h}}{\left(1+x_{h}\right)^{2}+\left(y_{h}+y_{c}\right)^{2}}
$$

where: $\alpha=1: x_{b}=1, y_{b}+y_{c}=0 . l$ corresponds to the length of the crimp cavity. The spiral structural layer can be adjusted to obtain the appropriate acoustic reactance by adjusting the spiral structure. On this basis, the diameter of the hole in the perforated plate can be adjusted to match its overall acoustic resistance with the air layer, and finally achieve perfect sound absorption (Li 2017):

$$
l=\frac{c S^{\prime}}{\omega S} \cot ^{-1} y_{h}
$$

where: $c$ is the propagation velocity of sound waves in air, which is $340 \mathrm{~m} \cdot \mathrm{s}^{-1}$ at room temperature; $S$ is the area of the incident side plate of sound wave, $S=\pi d^{2} / 4\left(\mathrm{~mm}^{2}\right) ; S^{\prime}$ is the cross sectional area of the dorsal cavity, $S^{\prime}=\mathrm{w}^{2}(\mathrm{~mm} 2)$; $l$ is the equivalent length of the crimped back cavity $(\mathrm{mm}) ; d$ is the diameter of the perforated plate $(\mathrm{mm}) ; w$ is the width of the groove $(\mathrm{mm})$.

\section{Specimen structure}

Based on the acoustic ultra-surface low-frequency perfect absorber, the spiral structural layer designed in this study is composed of spiral structure and perforated plate, and is adjusted by combining the theory (Ming-Ming et al. 2018) with the actual interior wall structure size. To test in a larger size, the spiral structural layer whether still have low frequency sound absorption effect, was compared with Helmholtz resonance structural layer, solid structural layer and air layer in traditional wall. The main test of the study is focused on the sound absorption coefficient of medium and low frequency, which used impedance tubes for testing. Due to the impedance tube suite in the low and medium frequency used fill a diameter of $100 \mathrm{~mm}$ diameter, specimen are designed (Fig. 1), and combination of concrete specimen are shown in Tab. 1. In Fig. 1, d is the external diameter of the specimen, $d=100 \mathrm{~mm} ; d_{1}$ and $d_{2}$ are the apertures of perforated plate 1 and 2 respectively, $d_{1}=16 \mathrm{~mm}, d_{2}=8 \mathrm{~mm} ; \mathrm{h}$ is the thickness of the structure, $h=20 \mathrm{~mm} ; h_{0}$ is the thickness of the cover plate, $\mathrm{h}_{0}=5 \mathrm{~mm}$; $\mathrm{b}$ is the edge width, $\mathrm{b}=5 \mathrm{~mm}$; $\mathrm{w}$ is the width of the groove, and $\mathrm{w}=15 \mathrm{~mm}$. The material of the structural layer (structure + cover) is ethoxyline resin, and the material parameters are shown in Tab. 2.

Tab. 1: Specimen combinations.

\begin{tabular}{|c|l|l|c|}
\hline \multicolumn{1}{|c|}{ Specimen } & \multicolumn{1}{|c|}{ Mark } & \multicolumn{1}{c|}{ Combination } & Quantity \\
\hline Spiral structural layer & M08 & Perforated panel 2 + Spiral structure & 3 \\
\cline { 2 - 4 } & M16 & Perforated panel 1 + Spiral structure & 3 \\
\hline
\end{tabular}




\begin{tabular}{|l|l|l|c|}
\hline \multirow{2}{*}{$\begin{array}{l}\text { Helmholtz resonance } \\
\text { structural layer }\end{array}$} & G08 & Perforated panel 2 + Cavity structure & 3 \\
\cline { 2 - 4 } & G16 & Perforated panel 1 + Cavity structure & 3 \\
\hline Air layer & K & Solid + Cavity structure & 3 \\
\hline Solid structural layer & S & Solid + Solid structure & 3 \\
\hline
\end{tabular}

*The solid structural layer $(S)$ is a combination of traditional structures (control group).

Tab. 2: Material parameters structural layer.

\begin{tabular}{|c|c|c|c|}
\hline Material & $\mathbf{E}(\mathbf{G P a})$ & $\mathbf{v}\left(\mathbf{m} \cdot \mathbf{s}^{\mathbf{1}}\right)$ & $\left.\rho \mathbf{( k g} \cdot \mathbf{m}^{\mathbf{3}}\right)$ \\
\hline Ethoxyline resin & 4.35 & 368 & 1180 \\
\hline
\end{tabular}

Based on the structural layer test, the structural layer is combined with the wall structure, as shown in Fig. 2. The wall structure from outside to inside are orderly arranged with structural layer, OSB, insulation cotton, and OSB (material parameters are shown in Tab. 3).

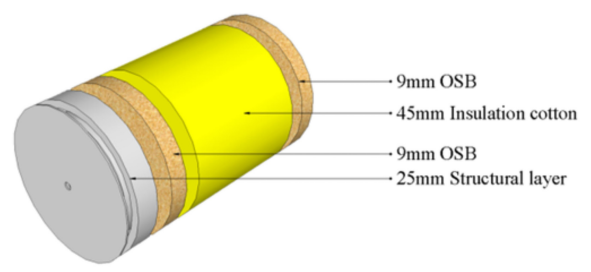

Fig. 2: Design drawing of composite wall.

Tab. 3: Wall material parameters.

\begin{tabular}{|l|c|c|}
\hline \multicolumn{1}{|c|}{ Material } & $\rho \mathbf{( k g \cdot \mathbf { m } ^ { - 3 } )}$ & $\mathbf{v}\left(\mathbf{m} \cdot \mathbf{s}^{\mathbf{- 1}}\right)$ \\
\hline Oriented strand board (OSB) & 593 & $1.8 \times 10^{3}$ \\
\hline
\end{tabular}

The specific wall combination is shown in Tab. 4, and the sound absorption coefficient of the wall structure is measured.

Tab. 4: Wall combination.

\begin{tabular}{|l|l|l|c|}
\hline \multicolumn{1}{|c|}{ Specimen } & \multicolumn{1}{c|}{ Mark } & \multicolumn{1}{c|}{ Combination (outside-inside) } & Quantity \\
\hline \multirow{2}{*}{ Spiral structural layer- Wall } & M08OBO & M08 + OSB + Insulated cotton + OSB & 3 \\
\cline { 2 - 5 } & M16OBO & M16 + OSB + Insulated cotton + OSB & 3 \\
\hline \multirow{2}{*}{ Helmholtz resonance structural layer- Wall } & G08OBO & G08 + OSB + Insulated cotton + OSB & 3 \\
\cline { 2 - 5 } & G16OBO & G16 + OSB + Insulated cotton + OSB & 3 \\
\hline Air layer- Wall & KOBO & K + OSB + Insulated cotton + OSB & 3 \\
\hline Solid structural layer- Wall & SOBO & S + OSB + Insulated cotton + OSB & 3 \\
\hline
\end{tabular}

*OBO is OSB (O), insulation cotton $(\mathrm{B})$, and OSB $(\mathrm{O})$. The solid structural layer- wall ( $\mathrm{SOBO})$ is a combination of traditional structures (control group).

\section{Methods}

Test methods

In this study, the impedance tube is used to measure the sound absorption coefficient, and the main principle is the transfer function method. The transfer function method is one 
of the common methods to measure the acoustic characteristics of materials and structures. The pipe and the dynamic acoustic characteristics of the specimen were used to measure the acoustic characteristics of the material structure (Zheng et al. 2018).

\section{Test procedures}

There were six sets of specimen combinations and six sets of wall combinations. Then three specimens in each group were tested repeatedly in three groups and averaged to output the corresponding sound absorption coefficient data at 150-1600 Hz. At last, OriginPro 2016 was used for data processing and analysis, and the test value curve of sound absorption coefficient was drawn. According to the curve drawing, the influence of spiral acoustic metasurface on the sound absorption of timber construction wall was investigated and the results were obtained.

\section{RESULTS AND DISCUSSION}

\section{Results}

Through theoretical calculation and experimental test, the theoretical and experimental values of sound absorption coefficient of spiral structural layer are obtained. The theoretical value is compared with the actual value to obtain the curve graph, as shown in Fig. 3. The acoustic absorption coefficient curves obtained by testing the six structural layers are shown in Fig. 4. Based on the sound absorption coefficient test of the structural layer, the structural layer is combined with the wall structure to measure the sound absorption coefficient curve of six wall structures, as shown in Fig. 5.

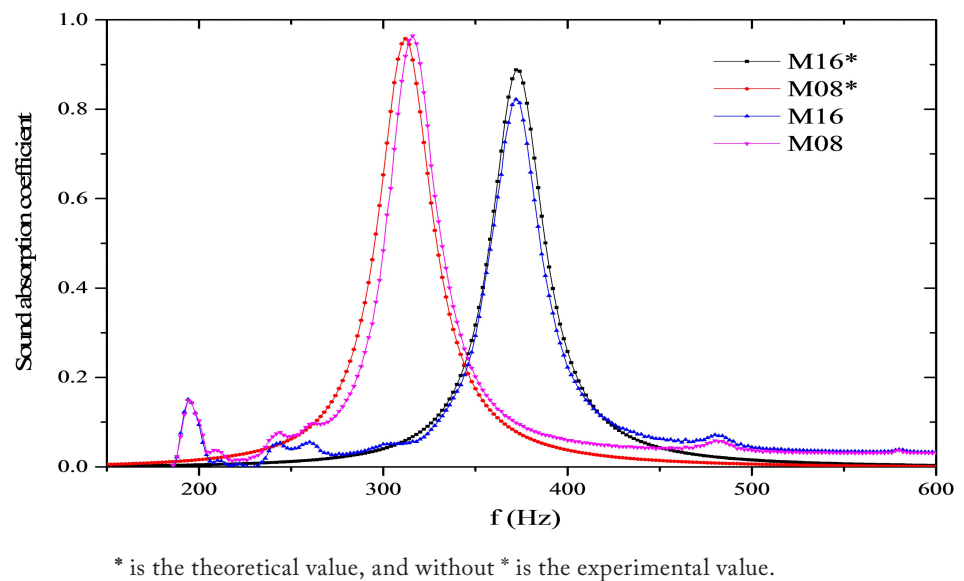

Fig. 3: Theoretical and experimental values of sound absorption coefficient of spiral structural layer.

According to the standards of ISO 354 (2003) and ISO 140-18 (2006), in the frequency range of $150-1600 \mathrm{~Hz}$, the average value obtained by calculating the sound absorption coefficient of the specimen is the average sound absorption coefficient, and the formula is expressed as Eq. 4. According to Eq. 4, the average sound absorption coefficients of six kinds of test piece structural layers and six kinds of wall structures are calculated, as shown in Tabs. 5 and 6 .

$$
\alpha=\left(\alpha_{160 \mathrm{~Hz}}+\alpha_{200 \mathrm{~Hz}}+\cdots+\alpha_{1600 \mathrm{~Hz}}\right) / 11
$$




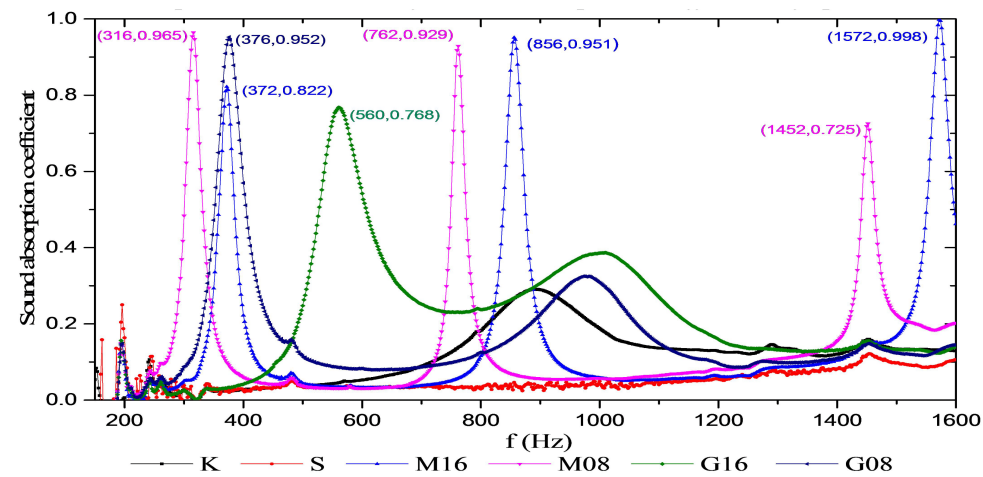

Fig. 4: Test value curves of sound absorption coefficient of six structural layers.

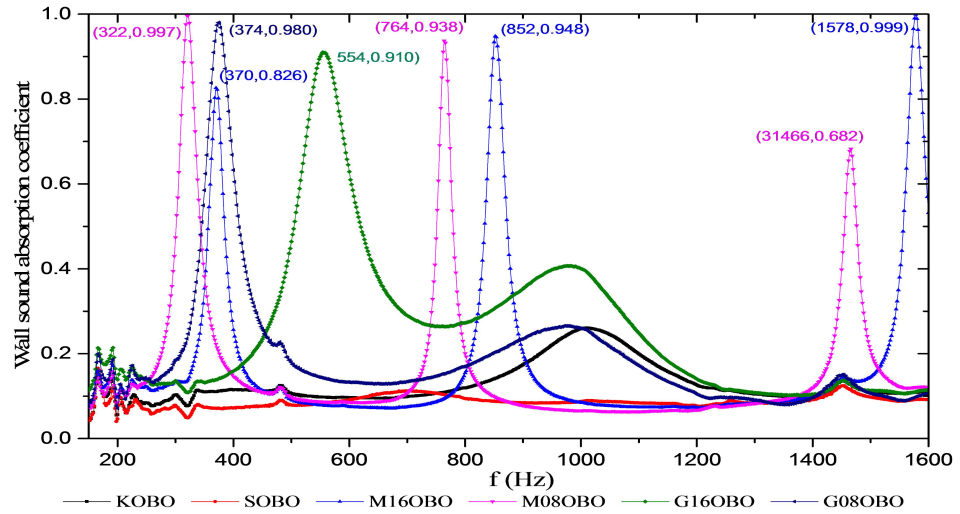

Fig. 5: Test value curves of sound absorption coefficient of six wall structures.

Tab. 5: Average sound absorption coefficient of the six structural layers.

\begin{tabular}{|c|c|c|c|c|c|c|}
\hline $\mathrm{f}(\mathrm{Hz}) \quad$ Combination & K & S & G08 & M08 & G16 & M16 \\
\hline 160 & 0.00 & 0.07 & 0.08 & 0.08 & 0.08 & 0.07 \\
\hline 200 & 0.01 & 0.16 & 0.10 & 0.10 & 0.10 & 0.09 \\
\hline 250 & 0.05 & 0.05 & 0.05 & 0.07 & 0.02 & 0.04 \\
\hline 316 & 0.00 & 0.01 & 0.12 & 0.96 & 0.01 & 0.06 \\
\hline 400 & 0.03 & 0.03 & 0.57 & 0.06 & 0.04 & 0.22 \\
\hline 500 & 0.04 & 0.03 & 0.12 & 0.04 & 0.25 & 0.04 \\
\hline 630 & 0.06 & 0.03 & 0.08 & 0.03 & 0.39 & 0.03 \\
\hline 800 & 0.18 & 0.03 & 0.13 & 0.16 & 0.24 & 0.12 \\
\hline 1000 & 0.19 & 0.05 & 0.31 & 0.06 & 0.39 & 0.06 \\
\hline 1250 & 0.12 & 0.06 & 0.09 & 0.09 & 0.14 & 0.06 \\
\hline 1600 & 0.13 & 0.11 & 0.15 & 0.20 & 0.14 & 0.46 \\
\hline $\begin{array}{c}\text { Average sound absorption } \\
\text { coefficient }\end{array}$ & 0.07 & 0.06 & 0.15 & 0.15 & 0.15 & 0.10 \\
\hline
\end{tabular}


Vol. 66 (3): 2021

Tab. 6: Average sound absorption coefficient of six wall structures.

\begin{tabular}{|c|c|c|c|c|c|c|}
\hline$f(\mathrm{~Hz}) \quad$ Combination & КОВO & SOBO & M16OBO & М08OBO & G16OBO & G08OBO \\
\hline 160 & 0.07 & 0.08 & 0.11 & 0.10 & 0.14 & 0.12 \\
\hline 200 & 0.06 & 0.05 & 0.10 & 0.09 & 0.14 & 0.07 \\
\hline 250 & 0.08 & 0.07 & 0.12 & 0.13 & 0.14 & 0.14 \\
\hline 316 & 0.08 & 0.05 & 0.13 & 0.94 & 0.11 & 0.22 \\
\hline 400 & 0.11 & 0.07 & 0.26 & 0.15 & 0.16 & 0.63 \\
\hline 500 & 0.11 & 0.08 & 0.09 & 0.10 & 0.46 & 0.18 \\
\hline 630 & 0.10 & 0.10 & 0.07 & 0.09 & 0.45 & 0.13 \\
\hline 800 & 0.12 & 0.09 & 0.15 & 0.20 & 0.27 & 0.17 \\
\hline 1000 & 0.26 & 0.09 & 0.08 & 0.07 & 0.40 & 0.26 \\
\hline 1250 & 0.11 & 0.08 & 0.08 & 0.08 & 0.12 & 0.10 \\
\hline 1600 & 0.11 & 0.09 & 0.53 & 0.12 & 0.11 & 0.11 \\
\hline $\begin{array}{c}\text { Average sound absorption } \\
\text { coefficient }\end{array}$ & 0.11 & 0.08 & 0.16 & 0.19 & 0.23 & 0.19 \\
\hline
\end{tabular}

\section{Analysis of sound absorption performance of structural layer}

It can be seen from Fig. 3 that the frequency of the first peak of the experimental value and the theoretical value is basically consistent. The influence of the change of perforated plate aperture on the sound absorption coefficient of the structural layer is also mutually satisfied by the experimental results and the theoretical results, but the numbers are slightly different. The possible reason is that both the sound field and external conditions designed in the theoretical calculation are ideal environments, while the effect of environmental sound waves during the field measurement is ignored. In addition, the materials used in the specimen are homogeneous materials in theoretical calculation, while there may be gaps in the structural layer during actual processing. In the case of sound absorption, the structural material itself may cause vibration, causing certain losses to sound waves. In general, the experimental results meet the theoretical results to a certain extent.

Based on the results obtained in Fig. 3, sound absorption coefficients of six structural layers of specimens were compared, as shown in Fig. 4. Within the frequency range of 150-1600 Hz, the sound absorption coefficient curve of the solid structural layer is gentle. The value does not exceed 0.2, and there is basically no peak value. Although the air layer structure has a peak value at about $850 \mathrm{~Hz}$, the peak value is only around 0.3 . In the whole frequency band, the sound absorption performance is not strong. According to Tab. 5, the average sound absorption coefficient of the solid structural layer and the air layer are $0.07,0.06$ respectively, indicating that the two structures basically do not absorb sound in the medium and low frequency bands. The reason for this phenomenon may be that the sound waves are transmitted to the solid structural layer, and most of the sound waves are reflected, while some medium and low frequency sound waves have strong penetrability, which cannot be absorbed by the solid structural layer, and the sound waves are transmitted. However, there is air impedance in the air layer; there is certain absorption of sound waves, but less consumption, especially for the medium and low frequency waves.

Compared with the solid structural layer and the air layer, the Helmholtz structural layer and the spiral structural layer have obvious sound absorption performance in the frequency range of 150-1600 Hz. According to Tab. 5, the average sound absorption coefficient of the two structural layers are within the range of 0.15 respectively. Compared with sound absorption 
performance of Helmholtz layer structure, spiral structural layer on the average absorption coefficient has no significant advantages. However, it can be seen from Fig. 3 that within the range of the test frequency band, there are three obvious peaks in the spiral structural layer. The three peaks occurred at about $350 \mathrm{~Hz}, 800 \mathrm{~Hz}$ and $1500 \mathrm{~Hz}$ respectively, and the peak sound absorption coefficient was between 0.71-1.0. The Helmholtz structure layer of perforated plate with the same aperture only has two peaks. The peak values are about $500 \mathrm{~Hz}$ and $1000 \mathrm{~Hz}$ respectively, and the peak values are smaller than the corresponding peak values in the spiral structural layer. The lower peak sound absorption coefficient is only 0.35 . In addition, it is found that compared with the first and second peaks of Helmholtz structural layer, the first and second peaks of spiral structural layer not only increase in value, but also move to low frequency and narrow the absorption band. This shows that the spiral structure does have a significant advantage in the absorption of medium and low frequency sound waves. The reason for this phenomenon is that airborne sound is a scalar wave and propagates freely without a cut-off frequency inside a waveguide with rigid walls. Because there is a large impedance difference between air and solid, the propagating path of the wave can be greatly extended by using coiling-up space with labyrinthine configuration (Assouar et al. 2018). Thus, with a longer propagating path from the coiling effect, the spiral structure has a lower resonant frequency and absorption peak. Compared with Helmholtz structure, the appropriate acoustic reactance can be obtained by adjusting the length of the spiral structure. On this basis, the diameter of the hole of the perforated plate can be adjusted to match the overall acoustic resistance with the air layer to achieve the perfect sound absorption in a certain frequency band.

At the same time, by comparing the spiral structural layers of different perforated plates, it was found that M08 with small aperture had three peaks of about $316 \mathrm{~Hz}, 762 \mathrm{~Hz}$ and $1452 \mathrm{~Hz}$ in the test frequency band. The sound absorption coefficients were 0.96, 0.93 and 0.72 , respectively, and the sound absorption coefficients were gradually decreasing. In the range of the test frequency band, the three peaks of M16 with larger aperture were around $372 \mathrm{~Hz}$, $856 \mathrm{~Hz}$ and $1572 \mathrm{~Hz}$, respectively. The sound absorption coefficients were $0.82,0.95$ and 0.99 , respectively. The sound absorption coefficient was gradually increasing, and the peak value moved towards high frequency as compared with M08. The main reason is that as the perforated plate aperture increases, the corresponding cross-sectional area of the hole also increases. However, the volume of the spiral air cavity structure does not change and the length of the holes does not change, so the peak value will move to the high frequency, which is determined by the resonance principle formula of Helmholtz sound absorption.

Therefore, compared with the traditional structure (solid structural layer and air layer), the spiral structure layer has obvious sound absorption performance in the range of 150$1600 \mathrm{~Hz}$. And the perforated plate aperture can affect the sound absorption performance of the spiral structure layer.

\section{Analysis of sound absorption performance of wall}

It can be seen from the comparison between Fig. 4 and Fig. 5 that the variation curve of sound absorption coefficient obtained by applying the structural layer to the wall structure is basically consistent with the trend and value of the curve of sound absorption coefficient, measured by the structural layer itself. This shows that changing the structural form of the structural layer can indeed optimize the sound absorption performance of the wall. In addition, the combination of the spiral structural layer and the wall structure can indeed optimize the sound absorption performance of the wall in the medium and low frequency band. Without reducing the overall sound absorption performance, it can achieve perfect sound absorption in some medium and low 
acoustic frequency bands.

At the same time, it can be roughly seen from Figs. 4, 5 and Tabs. 5, 6 that the peak value of sound absorption coefficient measured by the composite wall structure is basically consistent with the peak value of sound absorption coefficient of the structural layer in position, but slightly increased in value. According to the data in Tabs. 5 and 6, the comparison diagram of the average sound absorption coefficient of six groups of structural layers and walls are drawn (Fig. 6).

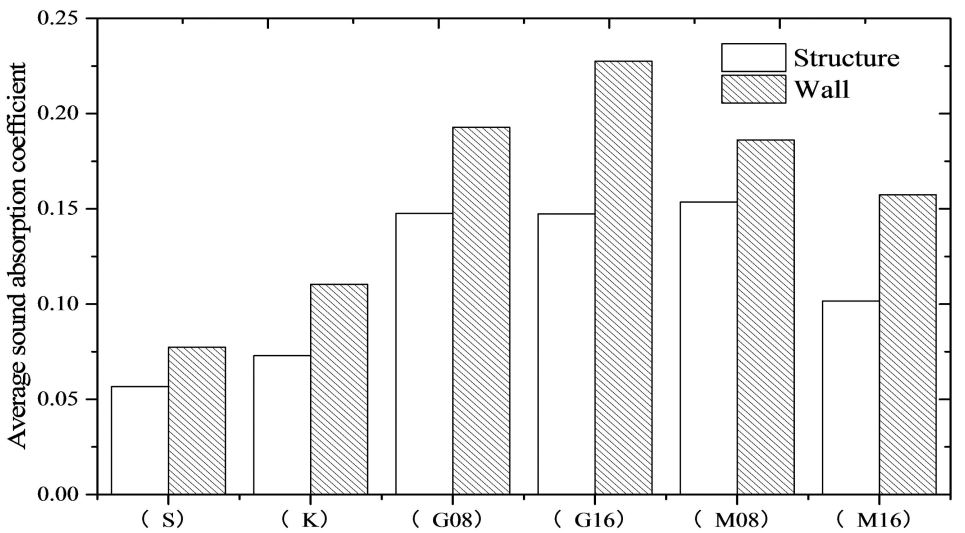

Fig. 6: Comparison of average sound absorption coefficient between six groups of structural layers and wall.

The average sound absorption coefficient measured by the wall composite structure are indeed higher than those measured by the structural layer. The main reason may be the absorption of sound waves by other structural materials in the wall, such as insulation cotton.

\section{CONCLUSIONS}

(1) The frequency of the first peak value of acoustic absorption coefficient of spiral structural layer is basically consistent with that of the theoretical value. (2) Analysis and comparison for six kinds of structural layer in the $150-1600 \mathrm{~Hz}$ frequency range of the absorption coefficient, the spiral structural layer had the best sound absorption performance in the test frequency range, which is obviously better than the traditional structure. (3) Within the range of the test frequency band, there were three obvious peaks in the spiral structural layer and the peak sound absorption coefficient was between 0.71-1.0. (4) The perforated plate aperture can affect the sound absorption performance of the spiral structure layer. (5) It shows that changing the structural form of the structural layer can optimize the sound-absorbing performance of the wall, especially in the medium and low frequency bands. (6) By using the acoustic spiral metasurface structure's efficient absorption performance of low-frequency sound waves and combining with the absorption performance of the timber construction wood wall's own absorption performance of medium-high frequency sound waves, the sound-absorbing and noise reduction performance of the timber construction wood wall, especially the low-frequency noise reduction performance, is effectively increased without affecting the mechanical and other physical properties of the wall. 


\section{ACKNOWLEDGMENT}

This study was funded by the Co-Innovation Center of Efficient Processing and Utilization of Forest Resources, Nanjing Forestry University, Nanjing 210037, China.

\section{REFERENCES}

1. Assouar, B., Liang, B., Wu, Y., Li, Y., Cheng, J.C., Jing, Y., 2018: Acoustic metasurfaces. Nature Reviews Materials (3): 460-472.

2. Ali, I., Mosa, A., Putra, R.R., Al-Ameri, E., 2020: Wideband sound absorption of a double-layer microperforated panel with inhomogeneous perforation. Applied Acoustics 161: 107167.

3. Azkorra, Z., Pérez, G., Coma, J., Luisa, F.C., 2015: Evaluation of green walls as a passive acoustic insulation system for buildings. Applied Acoustics 89: 46-56.

4. Bo-Seung, K., Sung-Jin, C., Dong-Ki, M., Park, J., 2016: Experimental study for improving sound absorption of a composite helical-shaped porous structure using carbon fiber. Composite Structures 145: 242-247.

5. Belakroum, R., Gherfi, A., Bouchema, K., Gharbi, A., 2017: Hygric buffer and acoustic absorption of new building insulation materials based on date palm fibers. Journal of Building Engineering 12: 132-139.

6. Caniato, M., Bettarello, F., Ferluga, A., Marsich, L., Schmid, C., Fausti, P., 2017: Acoustic of lightweight timber buildings: A review. Renewable and Sustainable Energy Reviews 80: 585-593.

7. Cao-ping, W., 2006: Study on sound absorption and sound insulation of gypsum board structure and its application. New Building Materials (8): 46-49.

8. Cheng, T., Hai-Tao, L., Dong-Dong, W., Rodolfo, L., Cong-Gan, Y., 2019: Mechanical performance of parallel bamboo strand lumber columns under axial compression: Experimental and numerical investigation. Construction and Building Materials 231: 117168.

9. Dah-You, M., 1998: Potential of microperforated panel absorber. Journal of Acoustical Society 104(5): 2861.

10. Hai-Tao, L., Gang, W., Zhen-Hua, X., Ileana, C., Ottavia, C., Xiao-Hong, X., HuiZhong, Z., Zhen-Yu, Q., 2019: Length and orientation direction effect on static bending properties of laminated Moso bamboo. European Journal of Wood and Wood Products 77(4): 547-557.

11. Huang, S., Fang, X., Wang, X., Badreddine, A., 2018: Acoustic perfect absorbers via spiral metasurfaces with embedded apertures. American Institute of Physics 113(2): 254-262.

12. Huang, S., Fang, X., Wang, X., Badreddine, A., 2019: Acoustic perfect absorbers via Helmholtz resonators with embedded apertures. Acoustical Society of America 145(1): 254-262.

13. Ingard, U., 1953: On the theory and design of acoustic resonators. The Journal of the Acoustical Society of America 25: 1037-1061.

14. ISO 354(E), 2003: Acoustics. Measurement of sound absorption in reverberation room.

15. ISO 140-18(E), 2006: Acoustics. Measurement of sound insulation in buildings and of building elements. Part 18: Laboratory measurement of sound generated by rainfall on building elements.

16. Krupali, D., Yi-Fan, Z., Shi-Wang, F., Li-Yun, C., 2019: Extreme low-frequency ultrathin acoustic absorbing metasurface. Applied Physics Letters 115: 173506. 
17. Ljunggren F., Ågren, A., 2011: Potential solutions to improved sound performance of volume based lightweight multi-storey timber buildings. Applied Acoustics 72(04): 231-240.

18. Li, Y., Assouar B.M., 2016: Acoustic metasurface-based perfect absorber with deep subwavelength thickness. Applied Physics Letters 108 (6): 063502.

19. Li, Y., 2017: Acoustic metasurfaces. Physical (11): 14-23.

20. Valachova, D., Skotnicova, I., 2019: Using the finite element method to predict heat dissipation in a timber frame building construction. Wood Research 64(5): 859-870.

21. Xin, L., Mahmud, A., Hai-Tao, L., Xiao-Yan, Z., Hong-Xu, W., Al-Deen, S., Paul, J.H., 2019: An experimental investigation on parallel bamboo strand lumber specimens under quasi static and impact loading. Construction and Building Materials 228: 116724.

22. Xin, L., Mahmud, A., Hai-Tao, L., Xiao-Yan, Z., Al-Deen, S., Hong-Xu, W., Paul, J.H., 2020: Experimental study on the deformation and failure mechanism of parallel bamboo strand lumber under drop-weight penetration impact. Construction and Building Materials 242: 118135.

23. Ying-Yang, L., Hai-Bei, X., Jia-Hua, K., 2018: Seismic evaluation of wood frame construction based on all connection deflection status. Wood Research 63(6): 979-992.

24. Zhong-Bin, X., Bai-Cun, W., San-Ming, Z., Rong-Jun, C., 2015: Design and acoustical performance investigation of sound absorption structurebased on plastic micro-capillary films. Applied Acoustics 89:152-158.

25. Zheng, W., Wen-Bo, X., Yao, L., Hai-Tao, Li., Zhi-Heng, W., Zhong, L., 2019: Dynamic and static testing methods for shear modulus of oriented strand board. Construction and Building Materials (216) 542-551

26. Zhu, Y.F., Donda, K., Fan, S.W., Cao, L.Y., Assouar, B., 2019: Broadband ultra-thin acoustic metasurface absorber with coiled structure. Applied Physics Express 12(11): 114002. 
Haiyan Fu, Xinyue Zhao

College Of Materials Science And Engineering

Nanjing Forestry University

2 IOO37 NANJING

ChINA

Patrick Adjei

College Of Civil Engineering

Nanjing Forestry University

2 IOO37 NANJING

China

$Z_{\text {HENG }}$ WANG $^{*}$

College Of Materials Science And Engineering

Nanjing Forestry University

2 IOOO37 NANJING

China

*Corresponding author: wangzheng63258@163.com

Xiaoli Wu

College Of Mechanical And Electrical

Nanjing Forestry University

2 IOO37 NANJING

China 